BMJ Open

Diabetes

Research

\& Care

\section{Efficacy and safety assessment of traditional Chinese medicine for metabolic syndrome}

To cite: Wu H, Tian J, Dai D, et al. Efficacy and safety assessment of traditional Chinese medicine for metabolic syndrome. BMJ Open Diab Res Care 2020;8:e001181. doi:10.1136/ bmjdrc-2020-001181

- Additional material is published online only. To view, please visit the journal online (http://dx.doi.org/10.1136/ bmjdrc-2020-001181).

Received 6 January 2020 Revised 13 February 2020 Accepted 18 February 2020

Check for updates

C) Author(s) (or their employer(s)) 2020. Re-use permitted under CC BY-NC. No commercial re-use. See rights and permissions. Published by BMJ.

'Department of Endocrinology, Guang'anmen Hospital, China Academy of Chinese Medical Sciences, Beijing, China

${ }^{2}$ Graduate College, Beijing University of Traditional Chinese Medicine, Beijing, China ${ }^{3}$ Department of National Integrated Traditional and Western Medicine Center for Cardiovascular Disease, ChinaJapan Friendship Hospital, Beijing, China

Correspondence to Dr Jiaxing Tian; tina_yai@126.com

Dr Fengmei Lian; Ifm565@sohu.com

Dr Xiaolin Tong; tongxiaolin@vip.163.com

\section{ABSTRACT}

Metabolic syndrome (MetS) is a multifarious metabolic disorder that could severely damage multiple organs. The emergence of MetS has markedly increased medical burden for patients. The treatment of MetS involves multitarget regulation, which is the advantage of traditional Chinese medicine (TCM). Many high-quality studies related to TCM for MetS have been conducted in recent years; however, no overall efficacy analysis has been reported. To evaluate the efficacy and safety of TCM against MetS, we reviewed randomized controlled trials of MetS published in the past decade and then selected and analyzed 16 highquality articles from over 800 papers. The results showed that TCM might be beneficial in improving body weight as well as in regulating glucose and lipid metabolisms; thus, TCM might be an ideal alternative therapy for MetS management. Treatment safety was also estimated in our analysis. A more elaborately designed and long-term observation of TCM for MetS should be performed in the future.

\section{INTRODUCTION}

Metabolic syndrome (MetS) is a cluster of risk factors often diagnosed by the co-occurrence of three out of the four following medical conditions: abdominal obesity, hypertension, hyperglycemia (diabetes mellitus (DM) or impaired glucose regulation) and dyslipidemia (hypertriglyceridemia or low highdensity lipoprotein (HDL) cholesterol). ${ }^{1}$ The prevalence of MetS in high-income countries is estimated to exceed $25 \%$ of the adult population. ${ }^{2}$ Moreover, the increased rate of MetS in low-incomed countries is dramatic. ${ }^{3} \mathrm{MetS}$ is regarded as a risk factor for the development of type 2 diabetes mellitus (T2DM) ${ }^{4}$ cardiovascular disease,${ }^{5}$ stroke,${ }^{6}$ polycystic ovary syndrome, non-alcoholic steatohepatitis ${ }^{7}$ and cancer, ${ }^{8}$ which may all lead to mortality. ${ }^{9}$ MetS pandemic may cause heavy social and economic burden. Thus, establishing an effective strategy to prevent MetS is urgent. MetS treatment involves an all-round approach aimed to retard weight gain, improve insulin resistance and dyslipidemia, and control blood glucose and blood pressure. ${ }^{10-16}$ Moreover, during treatment, the dosage of each drug must be evaluated because as the disease progresses, conventional agents often gradually lose their efficacy. Novel treatments for MetS, such as sodium glucose transporter-2 inhibitors, seem to be potential candidate drugs, but they need validation. ${ }^{17}$

For the treatment of MetS, traditional Chinese medicine (TCM) has a unique advantage owing to its holism concept and multitarget regulation. Several randomized controlled trials (RCTs) ${ }^{18-33}$ have shown the curative effect of TCM on MetS, with some studies focusing on the independent component of MetS. A multicenter, randomized, positive-controlled, $\mathrm{R}$ open-label clinical trial proved that the Chinese herb decoction Jiangtangtiaozhi (JTTZ) improves blood glucose and lipid metabolism, as well as reduces body weight. ${ }^{34}$ Another study also showed that JTTZ ameliorates T2DM with hyperlipidemia by enriching beneficial bacteria. ${ }^{35}$ Furthermore, TCM has been reported to be efficacious and safe for hyperlipidemia-associated diseases, as it mediates lipid metabolism disorders. ${ }^{36}$ Regarding obesity, TCM has been reported to be more effective than placebo or lifestyle modification in reducing body weight, showing similar efficacy but fewer side effects compared with antiobesity drugs. ${ }^{37}$ Other multicenter RCTs suggested that, compared with conventional pharmaceutical treatment, TCM as an add-on therapy exerts favorable effects on obesity-related hypertension. ${ }^{38}$ However, not all of the above-mentioned findings were obtained from a high-quality study with a sufficient number of participants or with subjects precisely diagnosed with MetS. Considering these limitations, we selected high-quality studies (according to the Jadad Scale) published in the past decade to evaluate the efficacy of TCM on MetS through an updated systematic review and meta-analysis. 


\section{MATERIALS AND METHODS}

\section{Search strategy and data sources}

This review was conducted under the guideline of the Preferred Reporting Items for Systematic Reviews and Meta-Analyses. Relevant clinical trials were identified by searching for papers published from January 2009 to December 2019 in the following databases: Web of Science, PubMed, Embase, MEDLINE, Cochrane Library, International Pharmaceutical Abstracts, Global Health, Sinomed, China National Knowledge Internet (CNKI), WanFang and VIP. Search terms included the following: ("metabolic syndrome" or "MetS" or "metabolism syndrome") and ("randomized controlled trial" or "controlled clinical trial" or "random" or "randomly" or "randomized" or "control" or "RCT") and ("TCM" or "traditional Chinese medicine" or "Chinese medicinal herb" or "Chinese herbal medicine" or "decoction" or "formula" or "prescription" or "Chinese patent medicine" or "Chinese patent drug" or "Chinese herbal compound prescription”).

\section{Study selection}

We included clinical trials that satisfied the following criteria: (1) studies where the participants had a definite diagnosis of MetS and were randomly assigned to receive TCM and Western medicine/placebo; (2) studies with a sample size of $\geq 60$; (3) studies with a treatment duration of $\geq 12$ weeks; (4) studies designed to focus on comparing TCM and Western medicine, or TCM and placebo; (5) studies that included efficacy evaluation on glycosylated hemoglobin (HbA1c), fasting plasma glucose (FPG) and 2-hour postprandial blood glucose (2hPG) for blood glucose; body mass index (BMI) and waist circumference (WC) for obesity; total cholesterol (TC), triglyceride (TG), low-density lipoprotein (LDL) cholesterol, and HDL for lipids; as well as systolic blood pressure (SBP) and diastolic blood pressure (DBP) for blood pressure; (6) studies that were designed to be RCTs; and (7) studies where methodological quality was evaluated for each study with the Jadad score from 4 to 7 (the strongest). ${ }^{39}$ We excluded clinical studies with the following features: (1) studies that were non-randomized, (2) studies where patients were enrolled with no definite diagnosis, (3) studies that reported only symptomatic changes in patients without laboratory indicators and (4) studies with a Jadad score of $<4$.

\section{Statistical analysis}

Data were analyzed using the RevMan V.5.3 software. ${ }^{40}$ Continuous outcomes were pooled for calculation of weighted mean differences accompanied by $95 \%$ CIs. Categorical outcomes were pooled for calculation of relative risks (RRs) accompanied by $95 \%$ CIs. $I^{2}$ statistics were used to measure heterogeneity. A fixed-effect (FE) model was used if $I^{2}$ was $<50 \%$; otherwise, the random-effect (RE) model was used. Publication bias was explored by means of funnel-plot analysis.

\section{RESULTS}

A total of 806 potentially relevant articles were identified during database searching (155 from CNKI, 239 from WanFang, 74 from VIP, 84 from Sinomed, 59 from Web of Science, 50 from PubMed, 50 from MEDLINE, 36 from Cochrane and 59 from Embase). Of these, 372 records were excluded because they were duplicates and 363 were excluded based on the titles and abstracts. A total of 71 articles were extracted from the second-round screening, and, finally, 16 articles remained after the full texts were assessed for eligibility: 14 in Chinese and 2 in English. A flow diagram of the screening is shown in online supplementary figure 1. In total, 1898 patients with MetS from 16 RCTs were included. All characteristics of the included RCTs are displayed in table 1, while the quality assessments and the pharmacological effects of TCM ingredients are summarized in online supplementary tables 1 and 2.

\section{TCM versus placebo}

Eleven RCTs that investigated TCM efficacy on MetS were analyzed. Sample sizes ranged from 60 to 169 patients, and the intervention duration was 12 weeks. The methodological quality of the studies was assessed by Jadad scores from 4 to 7 . The TCM interventions used for MetS included empirical decoctions and Chinese patent drugs combined with basic treatment, including health education, oral hypoglycemic agents, oral antihypertensive agents, and dyslipidemia drugs.

Results of obesity-related indicators indicated that TCM is more efficacious than placebo in reducing BMI $(\mathrm{n}=1123, \mathrm{RR}-1.01,95 \% \mathrm{CI}-1.71$ to 0.32 ; figure 1$)$ and that TCM decreases WC $(\mathrm{n}=851, \mathrm{RR}-1.65,95 \%$ CI -2.61 to 0.69 ; figure 2). Liu and $\mathrm{Cui}^{33}$ investigated the efficacy of Wenpi Fuzhen decoction in 70 patients with MetS. Patients in the treatment group lost more weight than those in the control group and showed decreases in $\mathrm{BMI}$ and WC compared with those in the control group $(\mathrm{p}<0.01$ and $\mathrm{p}<0.05)$. Wang et $a l^{25}$ enrolled 96 patients with MetS with T2DM. All patients were measured for BMI, $\mathrm{WC}$ and waist-to-hip ratio ( $\mathrm{WHtR}$ ) to assess obesity; the results showed that these parameters decreased significantly in the treatment group $(\mathrm{p}<0.01)$ with significant differences compared with the control group $(\mathrm{p}<0.05$ and $\mathrm{p}<0.01)$. The content of each component was also measured; it was shown that patients with central obesity had improved control with Yiqi Huaju formula $(p<0.01)$. In a multicenter, randomized, double-blind, parallelcontrolled trial, Wang ${ }^{31}$ found that the BMI of patients treated with Jiangtang pill significantly declined from $26.68 \pm 2.30$ to $23.41 \pm 2.16 \mathrm{~kg} / \mathrm{m}^{2}$ compared with that of those treated with placebo $(\mathrm{p}<0.01)$ (table 2$)$.

Regarding blood glucose indicators, the efficacy of TCM in reducing HbAlc is shown in figure $3(n=820, R R-0.25$, $95 \%$ CI -0.45 to 0.06 ). The hypoglycemic effect of TCM on FPG and 2hPG is shown in online supplementary figure 2 $(\mathrm{n}=1183, \mathrm{RR}-0.31,95 \%$ CI -0.40 to 0.22$)$ and in online supplementary figure 3 ( $\mathrm{n}=1062$, RR $-0.68,95 \% \mathrm{CI}-0.85$ to 


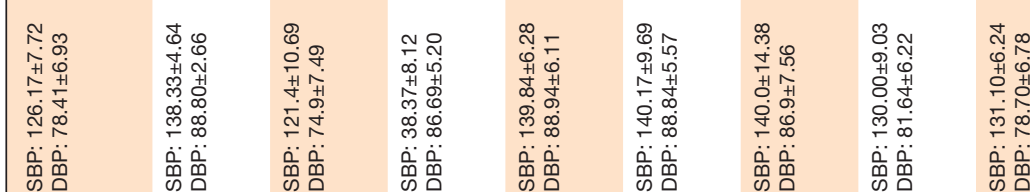

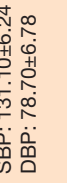

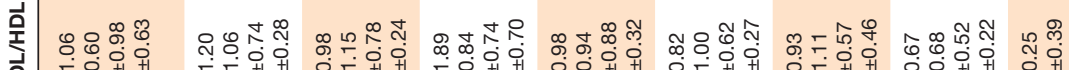

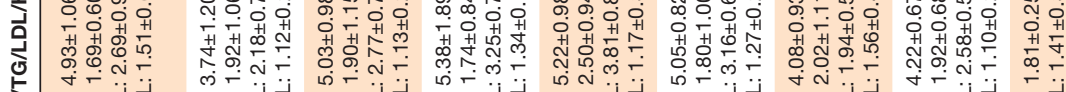

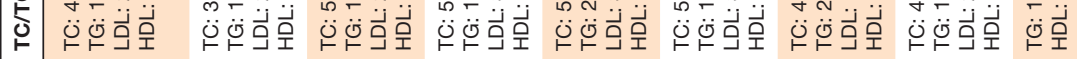

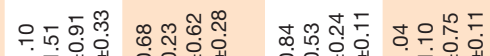

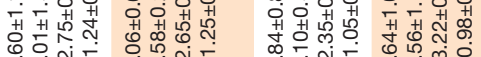

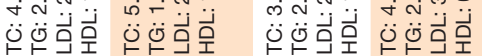

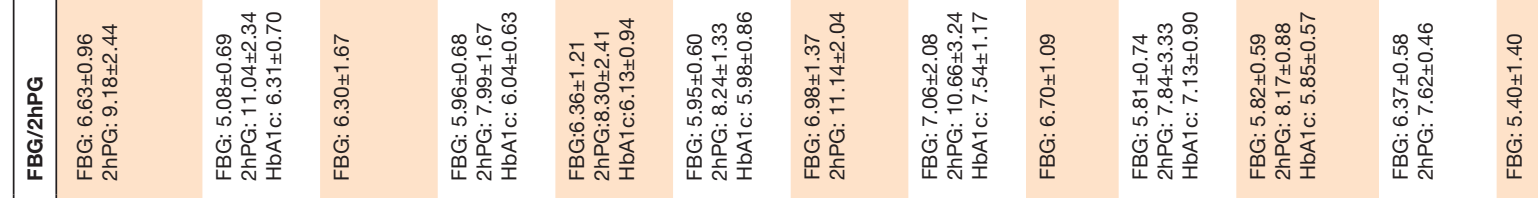

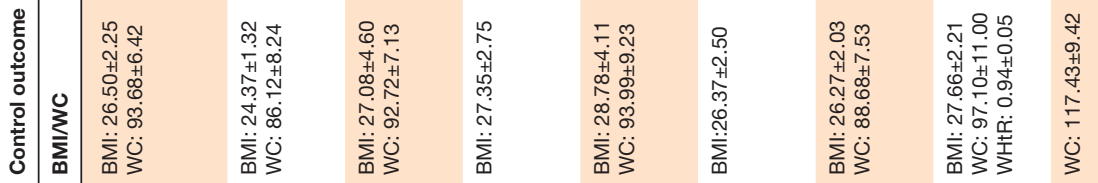

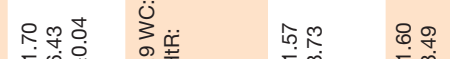

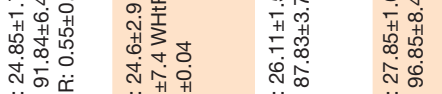

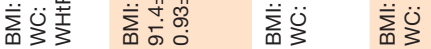

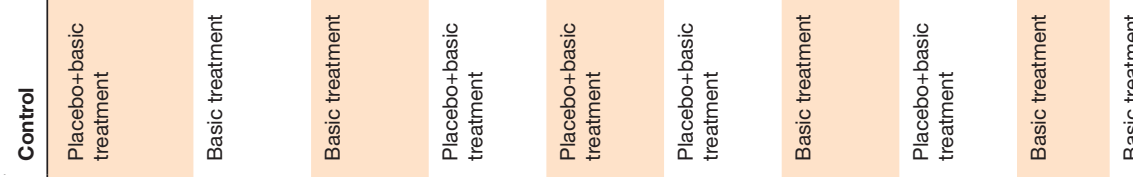

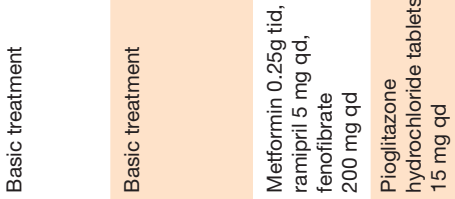

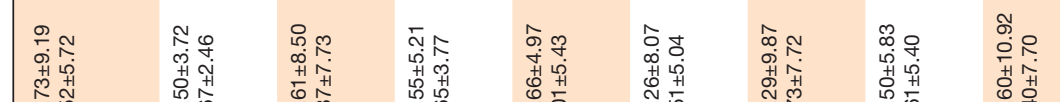

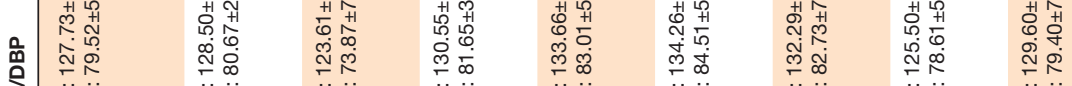

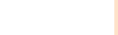

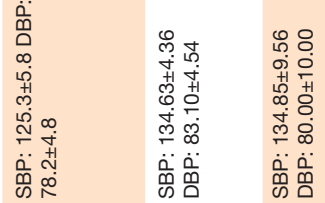

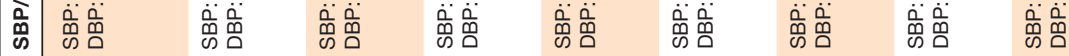

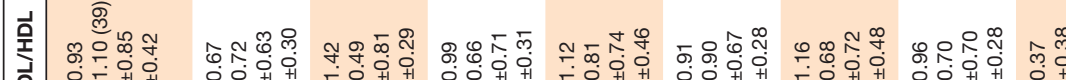

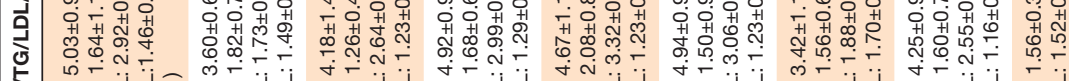

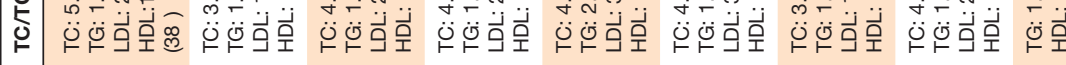

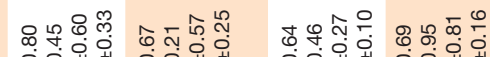

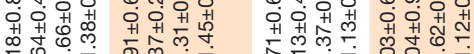

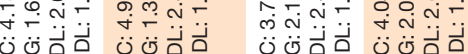

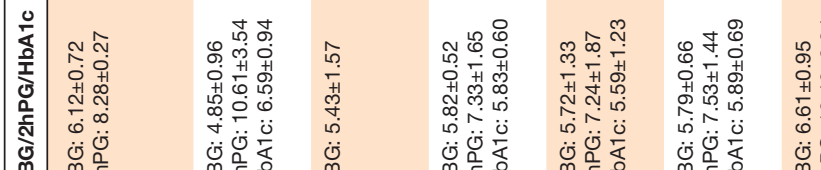

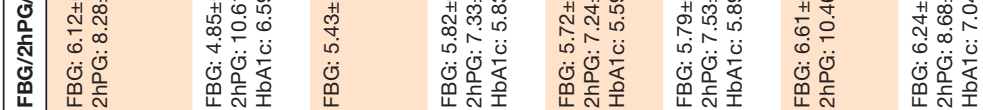

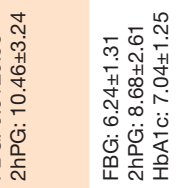

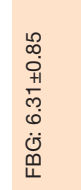

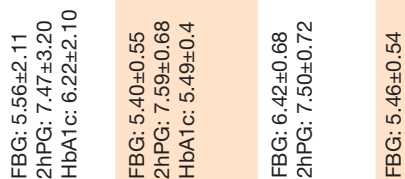

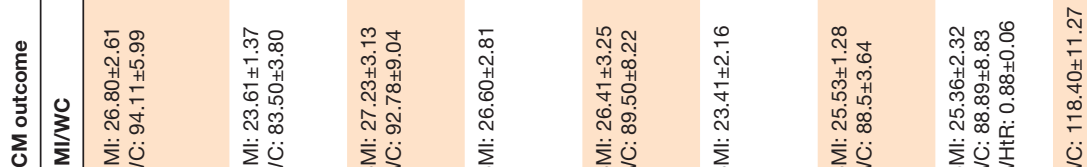

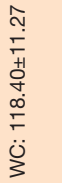

सतथ

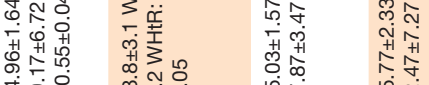

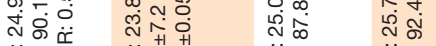

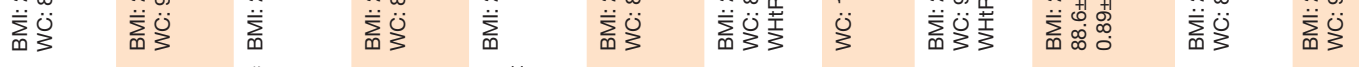

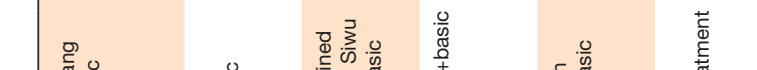

들

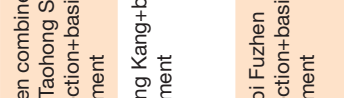

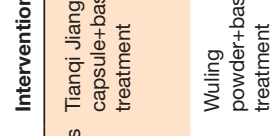

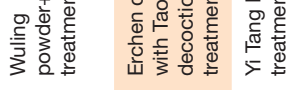

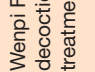

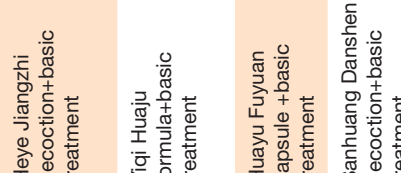

일 
0.50). Analysis of fasting insulin (FINS) revealed that TCM improved islet function ( $\mathrm{n}=343$, RR $-1.50,95 \%$ CI -2.17 to 0.83 ; online supplementary figure 4 ). The efficacy of TCM in improving insulin resistance was observed by homeostasis model assessment of insulin resistance (HOMA-IR) ( $\mathrm{n}=528, \mathrm{RR}-0.46,95 \%$ CI -0.68 to 0.25 ; online supplementary figure 5). In a multicenter RCT, ${ }^{20} 165$ patients with MetS with impaired glucose regulation (IGR) were treated with Yi Tang Kang or placebo. The results showed that Yi Tang Kang effectively lowered FPG and 2hPG $(p<0.01)$, especially $2 \mathrm{hPG}(\mathrm{p}<0.01)$. Another multicenter, doubleblind, placebo-control RCT $^{32}$ recruited 112 patients with MetS with impaired glucose tolerance (IGT) who received Tianqi Jiangtang capsule, which was recommended in the China Guideline for Type 2 Diabetes (2017); 55 IGT patients recovered after more than 3 months of treatment. FPG and 2hPG in the treatment group decreased after treatment with Tianqi Jiangtang capsule $(\mathrm{p}<0.05)$, and the efficacy of this TCM on 2hPG was more remarkable than that of the control $(p<0.05)$. Wang et al. $(2012)^{31}$ also enrolled patients with MetS with IGR who were treated with Jiangtang pill. The data indicated that Jiangtang pill effectively reduced $2 \mathrm{hPG}$, regardless of age difference $(\mathrm{p}<0.01)$. Wang et al. (2013) ${ }^{22}$ reported that Heye Jiangzhi Decoction decreased FINS $(\mathrm{p}<0.01)$ and improved HOMA-IR $(\mathrm{p}<0.05)$.

Analysis of blood lipid indicators revealed that TCM reduced cholesterol $(\mathrm{n}=1073$, RR $-0.27,95 \%$ CI -0.44 to 0.10 ; online supplementary figure 6 ) and diminished TG ( $\mathrm{n}=1133$, RR $-0.23,95 \%$ CI -0.28 to 0.17 ; figure 4$)$. Changes in LDL and HDL were also observed, suggesting that TCM decreased LDL ( $\mathrm{n}=1072$, RR $-0.19,95 \%$ CI -0.27 to 0.11 ; online supplementary figure 7 ) and increased HDL ( $\mathrm{n}=1132$; RR 0.10 ; 95\% CI 0.02, 0.18; online supplementary figure 8 ). Wang et al. $(2013)^{22}$ showed that Heye Jiangzhi Decoction decreased TC $(\mathrm{p}<0.01)$ and increased HDL $(p<0.05)$, with an efficacy rate of $85 \%$ in regulating blood lipids. Another survey ${ }^{23}$ reported that Erchen combined with Taohong Siwu Decoction showed preponderant efficacy in regulating TC, TG $(\mathrm{p}<0.01)$, and LDL $(p<0.05)$, but its effect on HDL was not different than that of placebo $(p>0.05)$. Fan et al $(2012)^{29}$ showed that a classical formula of Wuling Powder increased LDL to a greater degree compared with placebo $(\mathrm{p}<0.01)$. Zhang et al. $(2014)^{26}$ also showed that TCM decoction reduced TG and TC $(\mathrm{p}<0.05)$, indicating its potent efficacy in regulating lipid metabolism.

Analysis of blood pressure indicators revealed that TCM decreased SBP ( $\mathrm{n}=1110, \mathrm{RR}-4.54,95 \%$ CI -6.90 to 2.19; figure 5) and reduced DBP ( $n=1110, R R-3.65,95 \%$ CI -5.46 to 1.84; Online supplementary figure 9 ). Zhang et al. $(2016)^{30}$ treated patients with MetS with hypertension with two Chinese patent drugs, namely Yangyin Jiangya capsule and Jiangzhuo Quyu Granule, and their results showed significant decreases in SBP and DBP in the treatment group $(p<0.05)$. Another study ${ }^{24}$ investigating the Chinese patent drug Huayu Fuyuan capsule revealed its remarkable effect in regulating SBP $(p<0.05)$. 


\begin{tabular}{|c|c|c|c|c|c|c|c|c|}
\hline \multirow{2}{*}{ Study } & \multicolumn{3}{|l|}{ TCM } & \multicolumn{4}{|c|}{ Contol } & \multirow{2}{*}{ d Mean difference $(95 \% \mathrm{Cl})$} \\
\hline & Intervention & Mean $\pm \mathrm{SD}$ & No. of Subjects & & Intervention & Mean \pm SD & No. of Subjects & \\
\hline Total (95\% Cl) & & & 932 & 단 & & & 906 & $-1.07[-1.72,-0.43]$ \\
\hline \multicolumn{9}{|l|}{ TCM is Placebo } \\
\hline Chen 2011 & Tianqi Jiangtang capsule + basic treatment & $26.80 \pm 2.61$ & 63 & $=-1$ & Placebo+ basic treatment & $26.50 \pm 2.25$ & 59 & $0.30[-0.56,1.16]$ \\
\hline Fan 2012 & Wuling Powder + basic treatment & $23.61 \pm 1.37$ & 40 & = & basic treatment & $24.37 \pm 1.32$ & 40 & $-0.76[-1.35,-0.17]$ \\
\hline Huang 2019 & Erchen combined with Taohong Siwu Decoction + basic treatment & $27.23 \pm 3.13$ & 30 & $\mapsto-1$ & basic treatment & $27.08 \pm 4.60$ & 31 & $0.15[-1.82,2.12]$ \\
\hline Liu 2017 & Wenpi Fuchen Decoction+ basic treatment & $26.41 \pm 3.25$ & 35 & 1 & Placebo + basic treatment & $28.78 \pm 4.11$ & 35 & $-2.37[-4.11,-0.63]$ \\
\hline Wang 2012 & Jiangtang pill+ basic treatment & $23.41+2.16$ & 86 & E & Placebo+ basic treatment & $26.37 \pm 2.50$ & 81 & $-2.96[-3.67,-2.25]$ \\
\hline Wang 2013 & Heye Jiangzhi Decoction + basic treatment & $25.53+1.28$ & 60 & . & basic treatment & $26.27 \pm 2.03$ & 60 & $-0.74[-1.35,-0.13]$ \\
\hline Wang 2016 & Yiqi Huaju Formula + basic treatment & $25.36+2.32$ & 48 & 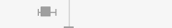 & Placebo+ basic treatment & $27.66+2.21$ & 48 & $-2.30[-3.21,-1.39]$ \\
\hline Zhang 2014 & Sanhuang Danshen Decoction+ basic treatment & $24.96 \pm 1.64$ & 36 & 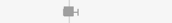 & basic treatment & $24.85+1.7$ & 37 & $0.11[-0.66,0.88]$ \\
\hline Zhang 2016 & Yangy in Jiangya capsule + Jiangzhuo Quyu Granule + basic treatment & $23.80 \pm 3.10$ & 85 & - & basic treatment & $24.60+2.90$ & 84 & $-0.80[-1.70,0.10]$ \\
\hline Chen 2014 & Heye Jiangzhi Decoction & $25.03 \pm 1.57$ & 30 & $=$ & Metformin,0.25g tid;Ramipril,5mg qd;Fenofibrate,200mg qd & $26.11 \pm 1.57$ & 30 & $-1.08[-1.87,-0.29]$ \\
\hline $\mathrm{Ji} 2017$ & Chaihu Sanren Decoction & $25.77 \pm 2.33$ & 33 & $=-1$ & Pioglitazone Hydrochloride Tablets, $15 \mathrm{mg}$ qd & $27.85+1.60$ & 33 & $-2.08[-3.04,-1.12]$ \\
\hline Li 2012 & Tang Zhi Ping & $24.43 \pm 0.51$ & 38 & - & Metformin, $0.25 \mathrm{~g}$ tid & $24.17 \pm 0.49$ & 37 & $0.26[0.03,0.49]$ \\
\hline Wang 2019 & Yiqi Huarhuo Gushen granules + basic treatment & $24.34 \pm 0.43$ & 50 & 룹 & Valsartan,80 mg qd + placebo + basic treatment & $27.61 \pm 2.75$ & 50 & $-3.27[-4.04,-2.50]$ \\
\hline Yu 2018 & Jiangtang Tiaozhi (JTTZ)formula & $27.33 \pm 3.34$ & 215 & E & Metformin tablets $0.25 \mathrm{~g}$ tid & $27.27 \pm 3.21$ & 199 & $0.06[-0.57,0.69]$ \\
\hline
\end{tabular}

Figure 1 Data summary of the high-quality RCTs of TCM interventions for BMI. BMI, body mass index; RCT, randomized controlled trial; TCM, traditional Chinese medicine.

Moreover, Yi Tang $\mathrm{Kang}^{20}$ led to notable improvement of blood pressure and decreased SBP and DBP $(\mathrm{p}<0.01)$.

\section{TCM versus Western medicine}

Five RCTs comparing the efficacy of TCM with that of Western medicine on MetS were analyzed. The sample sizes ranged from 60 to 414 patients and the treatment duration was 12 weeks. The methodological quality of the studies was assessed by Jadad scores of 4-6. In this comparison, two $\mathrm{RCTs}^{18}{ }^{27}$ used Chinese patent drugs as TCM intervention, whereas the others ${ }^{[19,21,28]}$ used empirical decoction. Three RCTs ${ }^{192728}$ used single oral hypoglycemic agents as the control group, one ${ }^{18}$ used antihypertensive drugs, and one RCT ${ }^{21}$ used a combination of oral hypoglycemic agents, oral antihypertensive drugs, and dyslipidemia drugs.

Analysis of obesity-related indicators showed no difference in efficacy between TCM and Western medicine in reducing BMI ( $\mathrm{n}=715$, RR $-1.19,95 \%$ CI -2.50 to 0.12 ; figure 1) and no significant difference between their efficacy in reducing WC $(\mathrm{n}=615, \mathrm{RR}-0.36,95 \%$ CI -1.44 to 0.71 ; figure 2). A total of 70 patients with $\mathrm{MetS}^{21}$ were assigned to the treatment group (Heye Jiangzhi Decoction) and the control group (Western medicine). After the 12-week treatment, the TCM group showed improvement of BMI compared with that in the metformin-treated group $(\mathrm{p}<0.05)$. A multicenter $\mathrm{RCT}^{18}$ evaluating the effect of Yiqi Huazhuo Gushen granules showed significant $(\mathrm{p}<0.05)$ differences in BMI and WHtR between the Yiqi Huazhuo Gushen-treated and valsartan-treated groups. $\mathrm{Ji}^{28}$ reported that Chaihu Sanren decoction ameliorated central obesity by reducing BMI $(\mathrm{p}<0.01)$ and WC $(\mathrm{p}<0.05)$, showing higher efficacy than that of pioglitazone hydrochloride tablets.

Analysis of blood glucose indicators revealed no remarkable difference in HbAlc between TCM and Western medicine, as shown in figure $3(n=589$, RR $-0.07,95 \%$ CI -0.31 to 0.18$)$. There were no differences in hypoglycemic effect on FPG $(n=715$, RR $-0.01,95 \%$ CI -0.40 to 0.39 ; Online supplementary figure 2 ) and $2 \mathrm{hPG}$ ( $\mathrm{n}=649, \mathrm{RR}-0.10,95 \%$ CI -0.32 to 0.13 ; online supplementary figure 3 ) between TCM and Western medicine. Moreover, there were no differences in effect on FINS $(\mathrm{n}=201, \mathrm{RR}-0.03,95 \%$ CI -0.46 to 0.40 ; Online supplementary figure 4$)$ and HOMA-IR ( $=715, \mathrm{RR}-0.06,95 \%$ CI -0.33 to 0.20 ; Online supplementary figure 5 ) between TCM and Western medicine. A multicenter, randomized, positive-controlled, open-label clinical trial ${ }^{19}$ indicated that the Chinese herbal formula JTTZ effectively improved blood glucose metabolism, reducing HbAlc by $0.75 \pm 1.32$ (95\% CI 0.58 to 0.93$)$, FPG by $1.4 \pm 2.4$ ( $\mathrm{p}<0.001), 2 \mathrm{hPG}$ by $2.42 \pm 4.53(\mathrm{p}<0.001)$ and HOMA-IR by $0.19 \pm 0.91(\mathrm{p}<0.01)$. In another study, 80 patients with MetS $^{27}$ were randomly assigned to the Tang Zhi Ping group or the metformin group, and the results showed that the Chinese patent drug exerted the same hypoglycemic effect as that of metformin. Furthermore, $\mathrm{Ji}^{28}$ reported that the Chaihu Sanren decoction had the same hypoglycemic efficacy in improving insulin resistance as that of pioglitazone hydrochloride $(p>0.05)$.

Analysis of blood lipid indicators showed that TCM was more effective than Western medicine in reducing TC $(\mathrm{n}=715$, RR $-0.35,95 \%$ CI -0.66 to 0.04 ; Online

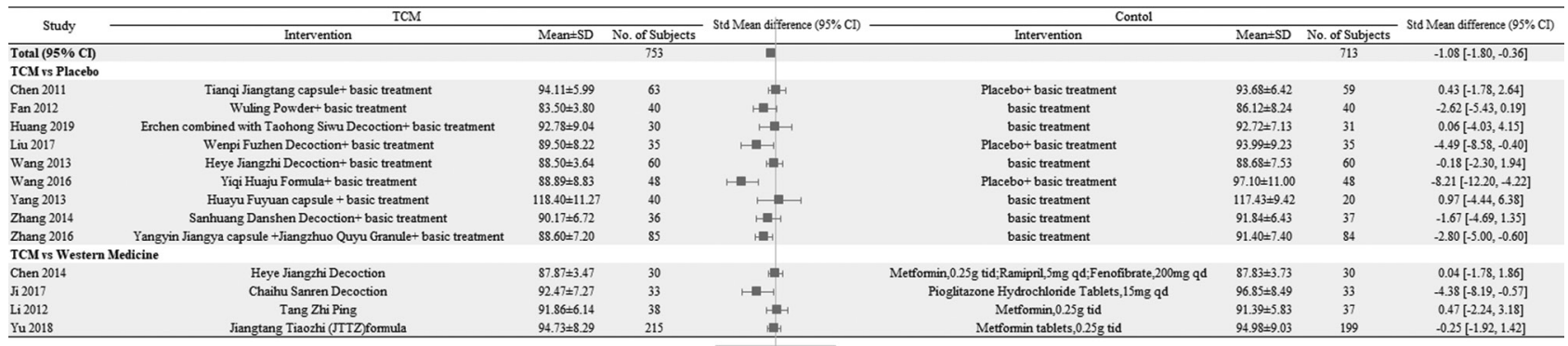

Figure 2 Data summary of the high-quality RCTs of TCM interventions for WC. RCT, randomized controlled trial; TCM, traditional Chinese medicine; WC, waist circumference. 


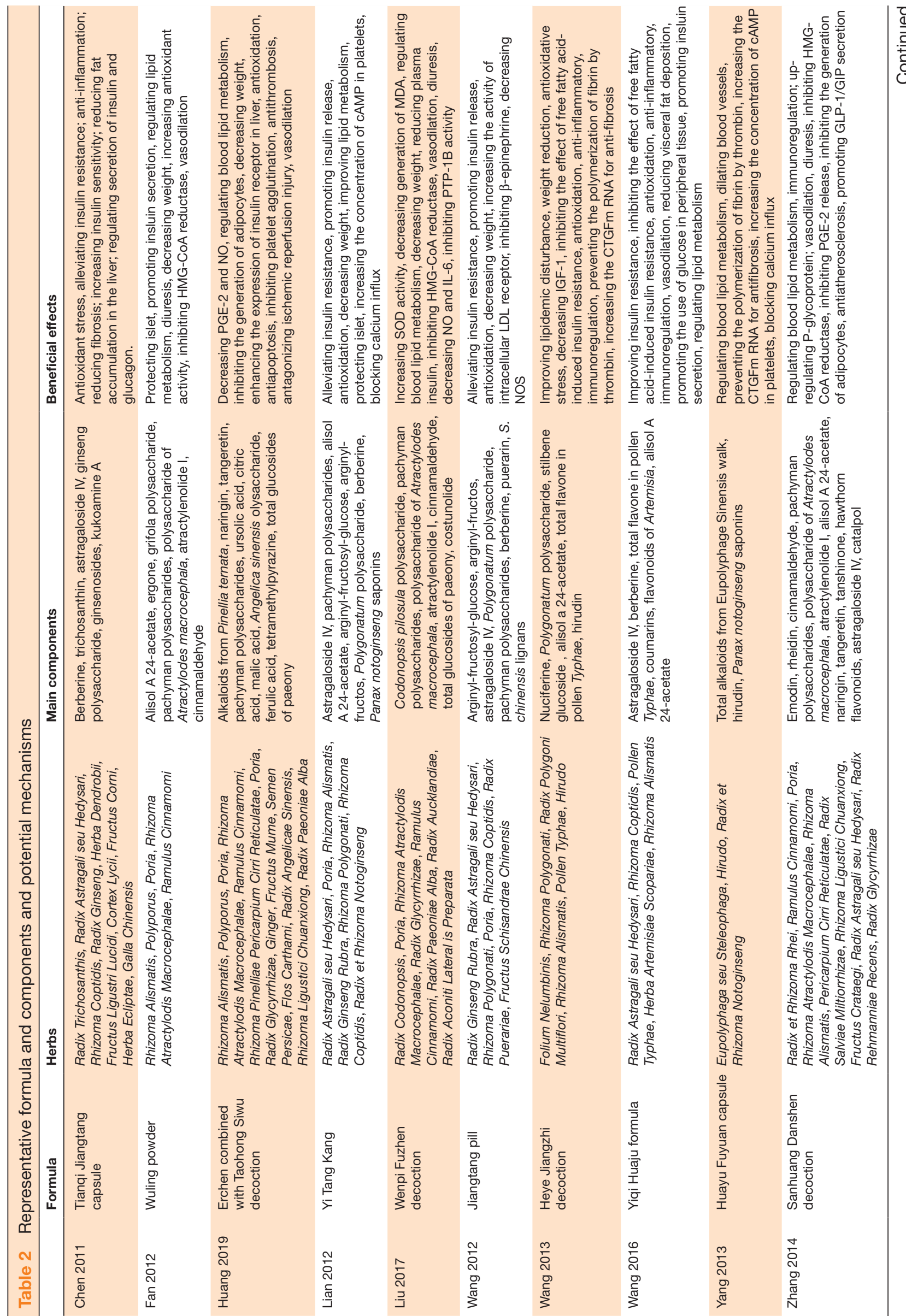




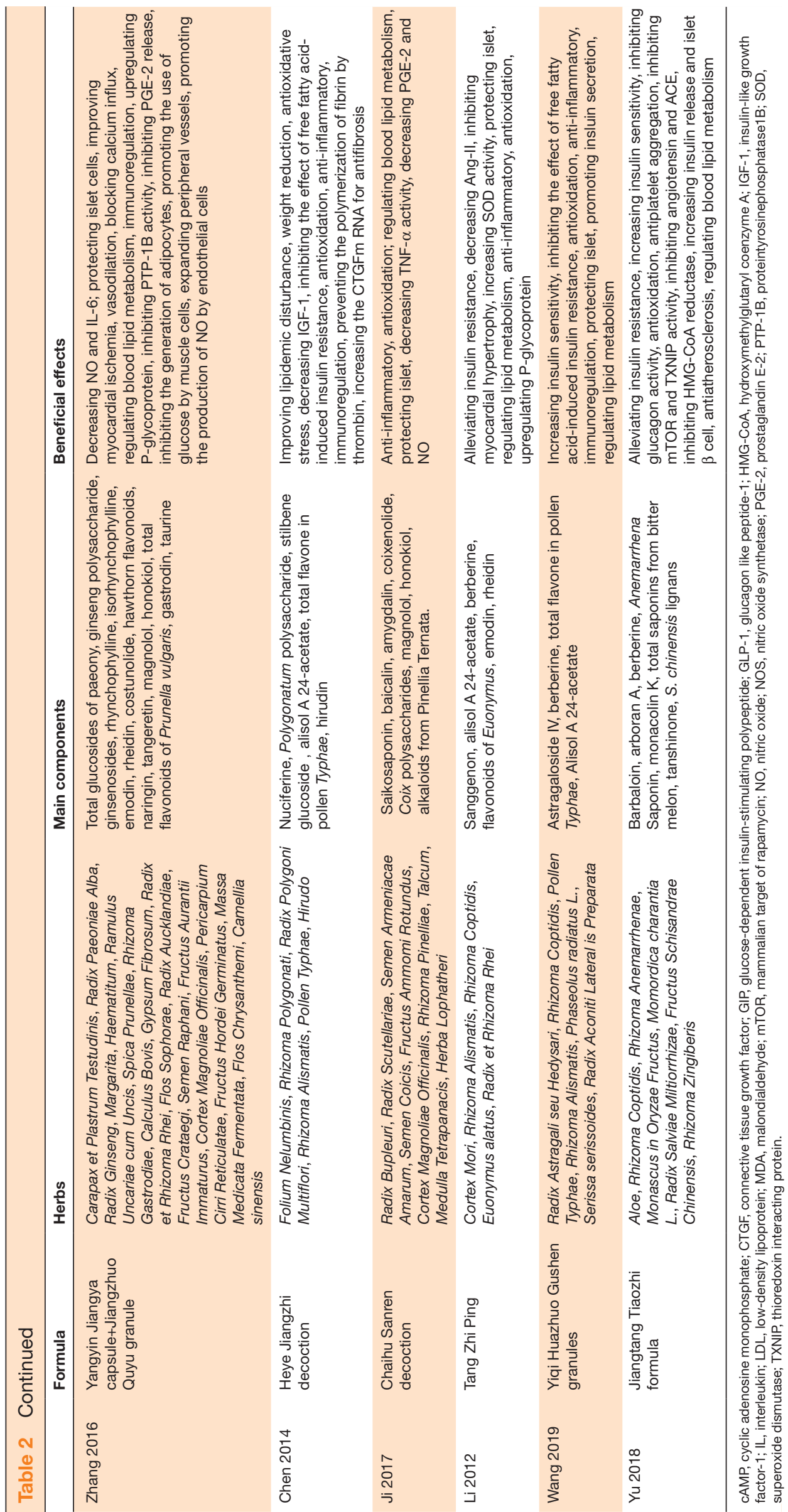




\begin{tabular}{|c|c|c|c|c|c|c|c|c|}
\hline \multirow[b]{2}{*}{ Study } & \multirow{2}{*}{\multicolumn{3}{|c|}{ TCM }} & \multirow{2}{*}{ Std Mean difference $(95 \% \mathrm{CD})$} & \multicolumn{3}{|l|}{ Contol } & \multirow[b]{2}{*}{ Std Mean differences $(95 \% \mathrm{CD})$} \\
\hline & & Mean \pm SD & No. of Subjects & & Intervention & Mean $\div$ SD & No. of Subjects & \\
\hline Total $(95 \% \mathrm{CI})$ & & & 716 & = & & & 693 & $-0.20[-0.36,-0.03]$ \\
\hline \multicolumn{9}{|l|}{ TCM rs Placebo } \\
\hline Fan 2012 & Wuling Powder t basic treatment & $6.59 \pm 0.94$ & 40 & 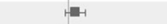 & basic treatment & $6.31 \pm 0.70$ & 40 & $0.28[-0.08,0.64]$ \\
\hline Lian 2012 & Yi Tang Kang+ basic treatment & $5.83 \pm 0.60$ & 83 & $=$ & Placebo+ basic treatment & $6.04 \pm 0.63$ & 82 & $-0.21[-0.40,-0.02]$ \\
\hline Liu 2017 & Wenpi Fuzhen Decoction+ basic treatment & $5.59 \pm 1.23$ & 35 & -1 & Placebo+ basic treatment & $6.13 \pm 0.94$ & 35 & $-0.54[-1.05,-0.03]$ \\
\hline Wang 2012 & Jiangtang pillt basic treatment & $5.89 \pm 0.69$ & 86 & $=$ & Placebo+ basic treatment & $5.98=0.86$ & 81 & $-0.09[-0.33,0.15]$ \\
\hline Wang 2016 & Yiqi Huaju Formula + basic treatment & $7.04 \pm 1.25$ & 36 & $=-$ & Placebo+ basic treatment & $7.54 \pm 1.17$ & 48 & $-0.50[-0.98,-0.02]$ \\
\hline Zhang 2014 & Sanhuang Danshen Decoction + basic treatment & $6.22 \pm 2.10$ & 85 & $\mapsto$ & basic treatment & $7.13 \pm 0.90$ & 37 & $-0.91[-1.65,-0.17]$ \\
\hline Zhang 2016 & Yangyin Jiangya capsule + Jiangzhuo Quyu Granule + basic treatment & $5.49 \pm 0.40$ & 85 & - & basic treatment & $5.85 \pm 0.57$ & 84 & $-0.36[-0.51,-0.21]$ \\
\hline \multicolumn{9}{|c|}{ TCM rs Western Medicine } \\
\hline Li 2012 & Tang Zhi Ping & $5.27 \pm 0.31$ & 38 & - & Metformin, $0.25 \mathrm{~g}$ tid & $5.19 \pm 0.48$ & 37 & $0.08[-0.10,0.26]$ \\
\hline Wang 2019 & Yiqi Huazhuo Gushen granules + basic treatment & $7.04+1.26$ & 50 & 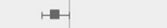 & Valsartan,80mg qd+placebo+basic treatment & $7.52 \pm 1.36$ & so & $-0.48[-0.99,0.03]$ \\
\hline Yu 2018 & Jiangtang Tiaozhi (JTZZ)formula & $7.51 \pm 1.44$ & 215 & - & Metfomin tablets, $0.25 \mathrm{~g}$ tid & $7.57 \pm 1.42$ & 199 & $-0.06[-0.34,0.22]$ \\
\hline
\end{tabular}

Figure 3 Data summary of the high-quality RCTs of TCM interventions for HbA1c. HbA1c, glycosylated hemoglobin; RCT, randomized controlled trial; TCM, traditional Chinese medicine.

supplementary figure 6). The TG-lowering effect of TCM was not different from that of Western medicine $(\mathrm{n}=715, \mathrm{RR}-0.53,95 \% \mathrm{CI}-0.64$ to 0.42 ; figure 4$)$. The outcome also showed that TCM was more effective in decreasing $\mathrm{LDL}(\mathrm{n}=715$, RR $-0.13,95 \% \mathrm{CI}-0.21$ to 0.04 ; Online supplementary figure 7) and in increasing HDL $(\mathrm{n}=301$, RR $0.08,95 \%$ CI 0.03 to 0.13 ; Online supplementary figure 8). A study showed that the Heye Jiangzhi decoction ${ }^{21}$ regulated lipid metabolism by affecting TC, TG, and LDL, but the effect showed minor difference from that of fenofibrate $(p>0.05)$. In addition, a better response on HDL was reported $(\mathrm{p}<0.05)$. Both $\mathrm{Yu}$ et $a l^{19}$ and $\mathrm{Li}$ et $a l^{27}$ used metformin as treatment for the control group; however, Li et al showed higher efficacy in reducing TC, TG and LDL $(\mathrm{p}<0.05)$, whereas Yu et al reported efficacy similar to that of metformin $(\mathrm{p}>0.05)$.

Analysis of blood pressure indicators showed no significant difference between the efficacies of TCM and Western medicine in decreasing SBP $(n=301, R R-3.81$, $95 \%$ CI -10.90 to 3.29 ; figure 5$)$ and DBP $(n=301$, RR $0.14,95 \%$ CI -3.21 to 3.50 ; Online supplementary figure 9). Wang $e t a l^{18}$ treated patients with MetS complicated with microalbuminuria (MAU) with Yiqi Huazhuo Gushen granules; the results showed that compared with valsartan, TCM exerted higher efficacy on SBP $(p<0.05)$ but similar efficacy on DBP ( $p>0.05)$.

\section{Publication bias}

A funnel plot was used to investigate potential publication bias. As shown in online supplementary figures 11-16, the symmetrical dispersion points suggested no publication bias in the comparison of TCM with the control group.

\section{DISCUSSION}

The findings described previously showed that TCM exerted efficacy on MetS, according to each studied parameter. Analysis of obesity indicators showed that TCM decreased body weight and WC, with efficacy similar to that of Western medicine. Blood glucose analysis showed that TCM, compared with placebo, exerted a DM-curative effect by lowering HbAlc, FPG, and 2hPG levels, as well as improving islet function and easing insulin resistance. TCM also improved lipid metabolism by reducing TC, TG, and LDL, as well as elevating HDL, with efficacy similar to that of Western medicine. Compared with placebo, TCM also exerted a blood pressure-controlling effect, although this effect was not significantly different from that of Western medicine.

In detail, TCM showed efficacy in decreasing body weight, although its efficacy in decreasing WC or central obesity was not significant. TCM showed significant efficacy in improving glucose metabolism compared with placebo, but this effect was not remarkable compared with that of Western medicine. Moreover, TCM exerted marked efficacy in improving lipid metabolism; it decreases TC, TG, and LDL levels and increases HDL level. However, the efficacy of TCM in controlling blood pressure was not notable. In summary, TCM might be more beneficial for decreasing body weight, as well as regulating lipid and glucose metabolisms for the treatment of MetS.

The first definition of MetS was proposed by the WHO in 1998, in which insulin resistance is regarded as the core factor. The WHO diagnosis criteria of MetS were

\begin{tabular}{|c|c|c|c|c|c|c|c|c|}
\hline \multirow{2}{*}{ Study } & \multicolumn{3}{|l|}{ TCM } & \multirow{2}{*}{ Std Mean difference $(95 \% \mathrm{CD})$} & \multicolumn{3}{|l|}{ Contol } & \multirow{2}{*}{ Std Mean differenece $(95 \% \mathrm{Cl})$} \\
\hline & Intervention & Mean $\pm S D$ & No. of Subjects & & Intervention & Mean \pm SD & No. of Subjects & \\
\hline \multirow{2}{*}{\multicolumn{9}{|c|}{ TCM rs Placebo }} \\
\hline & & & & & & & & \\
\hline Chen 2011 & Tianqi Jiangtang cap sule + basic treatment & $1.64 \pm 1.10$ & 39 & 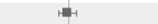 & Placebo + basic treatment & $1.69=0.60$ & 33 & $-0.05[-0.45,0.35]$ \\
\hline Fan 2012 & Wuling Powdert basic treatment & $1.82 \pm 0.72$ & 40 & $=$ & basic treatment & $1.92 \pm 1.06$ & 40 & $-0.10[-0.50,0.30]$ \\
\hline Huang 2019 & Erchen combined with Taohong Siruu Decoction- basic treatment & $1.26 \pm 0.49$ & 30 & $=$ & basic treatment & $1.90+1.15$ & 31 & $-0.64[-1.08,-0.20]$ \\
\hline Lian 2012 & Yi Tang Kang-basic treatment & $1.68 \pm 0.66$ & 83 & $=$ & Placebo+ basic treatment & $1.74 \pm 0.84$ & 82 & $-0.06[-0.29,0.17]$ \\
\hline Liu 2017 & Wenpi Furhen Decoction+ basic treatment & $2.08 \pm 0.81$ & 35 & $=$ & Placebo + basic treatment & $2.50 \pm 0.94$ & 35 & $-0.42[-0.83,-0.01]$ \\
\hline Wang 2012 & Jiangtang pillt basic treatment & $1.50 \pm 0.90$ & 86 & - & Placebo+ basic treatment & $1.80 \pm 1.00$ & 81 & $-0.30[-0.59,-0.01]$ \\
\hline Wang 2013 & Heye Jianghhi Decoctiont basic treatment & $1.56 \pm 0.68$ & 60 & $=$ & basic treatment & $2.02 \pm 1.11$ & 60 & $-0.46[-0.79,-0.13]$ \\
\hline Wang 2016 & Yiqi Huaju Fomula+ basic treatment & $1.60 \pm 0.70$ & 48 & - & Placebo+ basic treatment & $1.92 \pm 0.68$ & 48 & $-0.32[-0.60,-0.04]$ \\
\hline Yang 2013 & Huayu Fuyuan cap sule + basic treatment & $1.56 \pm 0.37$ & 40 & - & basic treatment & $1.81 \pm 0.25$ & 20 & $-0.25[-0.41,-0.09]$ \\
\hline Zhang 2014 & Sanhuang Danshen Decoction+ basic treatment & $1.64 \pm 0.45$ & 36 & $=$ & basic treatment & $2.01 \neq 1.51$ & 37 & $-0.37[-0.88,0.14]$ \\
\hline Zhang 2016 & Yangyin Jiangya capsule + +Jiangzhuo Quyu Granule+ basic treatment & $1.37 \pm 0.21$ & ss & m & basic treatment & $1.58 \pm 0.23$ & 84 & $-0.21[-0.28,-0.14]$ \\
\hline \multicolumn{9}{|c|}{ 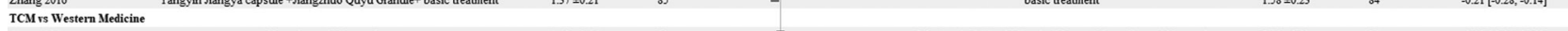 } \\
\hline Chen 2014 & Heye Jiangzhi Decoction & $2.13 \pm 0.46$ & 30 & - & Metformin, $0.25 \mathrm{~g}$ tid; Ramipril $5 \mathrm{mg}$ qd; Fenofibrate, $200 \mathrm{mg} \mathrm{qd}$ & $2.10 \pm 0.53$ & 30 & $0.03[-0.22,0.28]$ \\
\hline $\mathrm{Ji} 2017$ & Chaihu Sanren Decoction & $2.04 \pm 0.95$ & 33 & \pm & Pioglitazone Hydrochloride Tablets, $15 \mathrm{mg}$ qd & $2.56 \pm 1.10$ & 33 & $-0.52[-1.02,-0.02]$ \\
\hline Li 2012 & Tang Zhi Ping & $1.59 \pm 0.32$ & 38 & - & Metformin, $0.25 \mathrm{~g}$ tid & $2.75 \pm 0.46$ & 37 & $-1.16[-1.34,-0.98]$ \\
\hline Wang 2019 & Yiqi Huarhuo Gushen granules + basic treatment & $1.62 \pm 0.58$ & so & $=$ & Valsartan,80mg qd placebo obasic treatment & $1.88 \pm 0.44$ & so & $-0.26[-0.46,-0.06]$ \\
\hline Yu 2018 & Jiangtang Tiaozhi (JTTZ)formula & $2.79+1.86$ & 215 & $=$ & Metfomin tablets, $0.25 \mathrm{~g}$ tid & $2.82 \pm 1.91$ & 199 & $-0.03[-0.39,0.33]$ \\
\hline
\end{tabular}

Figure 4 Data summary of the high-quality RCTs of TCM interventions for TG. RCT, randomized controlled trial; TCM, traditional Chinese medicine; TG, triglyceride. 


\begin{tabular}{|c|c|c|c|c|c|c|c|c|c|}
\hline \multirow{2}{*}{ Study } & \multicolumn{3}{|l|}{$\mathrm{TCM}$} & \multirow{2}{*}{\multicolumn{2}{|c|}{ Std Mean difference $(95 \% \mathrm{CI})$}} & \multicolumn{3}{|l|}{ Contol } & \multirow{2}{*}{ Std Mean differences $(95 \% \mathrm{CI})$} \\
\hline & Intervention & Mean \pm SD & No. of Subjects & & & Intervention & Mean \pm SD & No. of Subjects & \\
\hline Total $(95 \% \mathrm{CI})$ & & & 721 & 팹 & & & & 690 & $-4.23[-6.86,-1.60]$ \\
\hline \multicolumn{10}{|l|}{ TCM rs Placebo } \\
\hline Chen 2011 & Tianqi Jiangtang capsule + basic treatment & $127.73 \pm 9.19$ & 63 & & 난 & Placebo+ basic treatment & $126.17 \pm 7.72$ & 59 & $1.56[-1.45,4.57]$ \\
\hline Fan 2012 & Wuling Powdert basic treatment & $128.50 \div 3.72$ & 40 & $=$ & & basic treatment & $138.33 \pm 4.64$ & 40 & $-9.83[-11.67,-7.99]$ \\
\hline Lian 2012 & $\mathrm{Y}_{\mathrm{i}}$ Tang Kang+ basic treatment & $130.55 \pm 5.21$ & 83 & - & & Placebo+basic treatment & $138.37 \pm 8.12$ & 82 & $-7.82[-9.90,-5.74]$ \\
\hline Liu 2017 & Wenpi Furhen Decoction + basic treatment & $133.66 \pm 4.97$ & 35 & 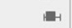 & & Placebo+basic treatment & $139.84 \pm 6.28$ & 35 & $-6.18[-8.83,-3.53]$ \\
\hline Wang 2012 & Jiangtang pillt basic treatment & $134.26 \pm 8.07$ & 86 & $=-1$ & & Placebo+basic treatment & $140.17 \pm 9.69$ & 81 & $-5.91[-8.62-3.20]$ \\
\hline Wang 2013 & Heye Jiangzhi Decoction+ basic treatment & $132.29=9.87$ & 60 & -1 & & basic treatment & $140.00=14.38$ & 60 & $-7.71[-12.12,-3.30]$ \\
\hline Wang 2016 & Yiqi Huaju Formula + basic treatment & $125.50 \pm 5.83$ & 48 & $=$ & & Placebo+ basic treatment & $130.00 \pm 9.03$ & 48 & $-4.50[-7.54,-1.46]$ \\
\hline Yang 2013 & Huayu Fuyuan capsule + basic treatment & $129.60 \pm 10.92$ & 40 & $=$ & $=$ & basic treatment & $131.10 \pm 6.24$ & 20 & $-1.50[-5.55,2.85]$ \\
\hline Zhang 2016 & Yangyin Jiangya capsule +Jiangzhuo Quyu Granule+ basic treatment & $125.30=5.80$ & 85 & - & & basic treatment & $128.80 \pm 6.30$ & \$4 & $-3.50[-5.33,-1.67]$ \\
\hline \multicolumn{10}{|c|}{ TCM rs Western Medicine } \\
\hline Chen 2014 & Heye Jiangzhi Decoction & $134.63 \pm 4.36$ & 30 & & - & Metformin $0.25 \mathrm{~g}$ tid;Ramipril $5 \mathrm{mg}$ qd; Fenofibrate, $200 \mathrm{mg}$ qd & $129.87 \pm 3.31$ & 30 & $4.76[2.80,6.72]$ \\
\hline $\mathrm{Ji}_{2} 2017$ & Chaihu Sanren Decoction & $134.85=9.56$ & 33 & $=-1$ & & Pioglitazone Hydrochloride Tablets, $15 \mathrm{mg}$ qd & $141.48 \pm 9.32$ & 33 & $-6.63[-11.19,-2.07]$ \\
\hline Li 2012 & Tang Zhi Ping & $127.22 \pm 8.13$ & 38 & $=$ & & Metfomin $0.25 \mathrm{~g}$ tid & $136.47 \pm 8.96$ & 37 & $-9.25[-13.13,-5.37]$ \\
\hline Wang 2019 & Yiqi Huarhuo Gushen granules + basic treatment & $125.91 \pm 7.12$ & so & $=$ & & Valsartan, $80 \mathrm{mg}$ qd placebo+basic treatment & $130.56 \pm 7.99$ & so & $-4.65[-7.62,-1.68]$ \\
\hline
\end{tabular}

Figure 5 Data summary of the high-quality RCTs of TCM interventions for SBP. RCT, randomized controlled trial; SBP, systolic blood pressure; TCM, traditional Chinese medicine.

formulated on the basis of IGR, DM, or insulin resistance combined with two additional factors, including increased arterial pressure, central obesity, increased plasma TG or decreased HDL, and MAU. ${ }^{41}$ In 1999, the European Group for Study of Insulin Resistance thought the definition should include the presence of insulin resistance or fasting hyperinsulinemia, as well as two of the following conditions: hypertension, dyslipidemia, and central obesity. ${ }^{42}$ Subsequently, the American National Cholesterol Education Programme Adult Treatment Panel III (ATP III) programme was launched in 2001, establishing criteria that included the presence of three of the following five factors: abdominal obesity, elevated TG, reduced HDL, hypertension, and elevated fasting glucose. ${ }^{43}$ Subsequently, the American Association of Clinical Endocrinologists recommended four components of the ATP III standard except WC and emphasized the importance of clinical symptoms. ${ }^{44}$ The International Diabetes Federation considered central obesity as the primary risk factor, combined with any two of the following conditions: elevated TG, reduced HDL, elevated blood pressure, elevated FPG, and previous diagnosis of T2DM. ${ }^{45}$ Considering the differences between these definitions, the transition from insulin resistance to abdominal obesity might provide insights into the mechanism of MetS.

The Chinese Diabetes Society recommended three or all of the following factors as diagnosis criteria: overweightness or obesity, elevated blood glucose (including FPG or 2hPG), hypertension, and dyslipidemia (including elevated TG, reduced HDL). ${ }^{41}$ In 2007 , a joint committee of Chinese experts in endocrinology, cardiology, diabetes, and docimasiology released a guideline that proposed the criteria as three or more of the following characteristics: central obesity, elevated TG, reduced HDL, hypertension, elevated blood glucose, and history of diabetes. ${ }^{46}$ Though there are differences among these definitions, the definitions are much more suitable for clinical use and for each region.

In our enrolled studies, patients were diagnosed with MetS according to different definitions. Yu et $a l^{19}$ observed the clinical efficacy of TCM on T2DM in terms of obesity and hyperlipidemia, according to the standard of Chinese Diabetes Society. ${ }^{47}$ The participants of three trials $^{20} 3132$ were diagnosed with MetS with IGR; in one trial, ${ }^{30}$ patients were diagnosed with MetS with hypertension; in another trial, patients were diagnosed with MetS with MAU. Wang et al ${ }^{18}$ observed the effect of TCM on MetS complicated with MAU. They used a combination of Yiqi Huazhuo Gushen granules and valsartan for 12 weeks and found that the combination showed improved efficacy against MAU $(\mathrm{p}<0.05)$. Though MAU was not a primary indicator, TCM was shown to decrease urinary microalbumin and delay the progression of MetS. The study also emphasized that attention should be paid to TCM as a secondary and tertiary preventive agent against MetS, as MetS can have various complications.

The RE and FE models were used for sensitivity analysis of the stability of the included studies. As the accurate numbers of each component in every study and the agents used for basic treatment were not provided in detail, we could not measure the effect of these factors on heterogeneity. In addition, different diagnostic criteria and complications might also cause heterogeneity. Moreover, the agents used as control might cause heterogeneity, as Wang $e a^{18}$ used valsartan; Li $e t a l^{27}$ used metformin; and Chen $^{21}$ used metformin, ramipril, and fenofibrate. Furthermore, Wang et alincluded patients with MetS with MAU, which might lead to heterogeneity. The patients in some studies ${ }^{2021} 23$ 25-28 32 were also selected according to TCM syndrome differentiation and different syndrome patterns. The use of empirical decoction or Chinese patent drugs according to the syndrome differentiation might also cause heterogeneity.

The incidence of adverse events (AEs) was evaluated to assess the safety of TCM. Twelve studies reported AEs and four ${ }^{180-32}$ did not mention any AE. The incidence of AEs in the TCM and control groups was not significantly different (RR 0.66, 95\% CI 0.40 to 1.08; Online supplementary figure 10 ), suggesting that TCM was generally safe. Details on the AEs are shown in Online supplementary table 2. The main $\mathrm{AE}$ observed in treatment group was diarrhea, which might be caused by Rheum. Only one patient ${ }^{26}$ dropped out of the study owing to diarrhea. Because this symptom did not last long and relative examinations showed kidney or liver injury, TCM as a treatment of MetS was considered safe to a certain degree.

A prominent limitation of the present study was the lack of high-quality RCTs. Though we included studies 
with high Jadad scores, there were four RCTs with a Jadad score of $6^{18} 203133$ and only one RCT had a Jadad score of $7 .^{32}$ The quality of the included studies would directly and seriously affect the accuracy of a metaanalysis. Besides, the duration of these RCTs was not long enough to provide a strong evidence, and we could not access the long-term efficacy of TCM on MetS-induced complications; future studies should focus on secondary and tertiary preventions. The efficacy of TCM on MetS might be due to weight loss or improvement of insulin sensitivity from the data mentioned previously. However, there is no definite pathological mechanism yet as MetS is the multifactorial disease. We have no idea which factor would accelerate the disease progression faster and the key pathway of treatment. The long-term observation of MetS and each single-factor study might offer references on exploring the mechanism. Regarding the participants, only one $\mathrm{RCT}^{19}$ included more than 200 patients in each group; thus, the efficacy and the ratio of AEs might be affected by the number of participants. Furthermore, though two RCTs ${ }^{18} 19$ were in English, all the participants were Chinese. Thus, the lack of other ethnic groups in the included studies might restrict the scope of their application. Data on cardiovascular events, degree of fatty liver, measurement of MAU, and detailed AEs may provide more persuasive evidence in future meta-analyses.

Contributors HW and JT drafted the manuscript. FL and XT revised the manuscript. DD, JL, XWa and XWe searched the literature and extracted data. DJ and XA made statistical analysis of data. All of the authors participated in the design and approved the final manuscript.

Funding This work was partially supported by the Young Elite Scientists Sponsorship Program by CAST (YESS20170034, 2018QNRC2-C10), the National Natural Science Foundation of China (numbers 81904187, 81274000 and 81803923), Special Scientific Research for Traditional Chinese Medicine of China (201507001-11) and the Outstanding Young Scientific and Technological Talents Program (ZZ13-YQ-026).

Competing interests None declared.

Patient consent for publication Not required.

Provenance and peer review Not commissioned; externally peer reviewed.

Data availability statement All data relevant to the study are included in the article or uploaded as supplementary information.

Open access This is an open access article distributed in accordance with the Creative Commons Attribution Non Commercial (CC BY-NC 4.0) license, which permits others to distribute, remix, adapt, build upon this work non-commercially, and license their derivative works on different terms, provided the original work is properly cited, appropriate credit is given, any changes made indicated, and the use is non-commercial. See: http://creativecommons.org/licenses/by-nc/4.0/.

ORCID iDs

Haoran Wu http://orcid.org/0000-0003-2906-510X

Jiaxing Tian http://orcid.org/0000-0002-1473-8474

Xuedong An http://orcid.org/0000-0002-2787-1645

\section{REFERENCES}

1 Chinese Diabetes Society. Standards of medical care for type 2 diabetes in China 2017. Chinese Journal of Practical Internal Medicine 2018;38:292-344.

2 Miller JM, Kaylor MB, Johannsson M, et al. Prevalence of metabolic syndrome and individual criterion in US adolescents: 2001-2010 National health and nutrition examination survey. Metab Syndr Relat Disord 2014;12:527-32.
3 Li W, Song F, Wang X, et al. Prevalence of metabolic syndrome among middle-aged and elderly adults in China: current status and temporal trends. Ann Med 2018;50:345-53.

4 Ramírez-Vélez R, Pérez-Sousa Miguel Ángel, González-Ruíz K, et al. Obesity- and Lipid-Related parameters in the identification of older adults with a high risk of prediabetes according to the American diabetes association: an analysis of the 2015 health, well-being, and aging study. Nutrients 2019;11:E2654.

5 Tune JD, Goodwill AG, Sassoon DJ, et al. Cardiovascular consequences of metabolic syndrome. Transl Res 2017;183:57-70.

6 Rodriguez-Colon SM, Mo J, Duan Y, et al. Metabolic syndrome clusters and the risk of incident stroke: the Atherosclerosis risk in communities (ARIC) study. Stroke 2009;40:200-5.

7 Mendrick DL, Diehl AM, Topor LS, et al. Metabolic syndrome and associated diseases: from the bench to the clinic. Toxicol Sci 2018;162:36-42.

8 Esposito K, Chiodini P, Colao A, et al. Metabolic syndrome and risk of cancer: a systematic review and meta-analysis. Diabetes Care 2012;35:2402-11.

9 Isomaa B, Almgren P, Tuomi T, et al. Cardiovascular morbidity and mortality associated with the metabolic syndrome. Diabetes Care 2001;24:683-9.

10 Dommermuth R, Ewing K. Metabolic syndrome: systems thinking in heart disease. Prim Care 2018;45:109-29.

11 Wei H, Liu S, Liao Y, et al. A systematic review of the medicinal potential of mulberry in treating diabetes mellitus. Am J Chin Med 2018;46:1743-70.

$12 \mathrm{Ng} \mathrm{K-W}$, Cao Z-J, Chen H-B, et al. Oolong tea: a critical review of processing methods, chemical composition, health effects, and risk. Crit Rev Food Sci Nutr 2018;58:2957-80.

13 Tang Y-N, He X-C, Ye M, et al. Cardioprotective effect of total saponins from three medicinal species of Dioscorea against isoprenaline-induced myocardial ischemia. J Ethnopharmacol 2015;175:451-5.

14 Guan X-R, Zhu L, Xiao Z-G, et al. Bioactivity, toxicity and detoxification assessment of Dioscorea bulbifera L.: a comprehensive review. Phytochemistry Reviews 2017;16:573-601.

15 Feng J-F, Tang Y-N, Ji H, et al. Biotransformation of Dioscorea nipponica by Rat Intestinal Microflora and Cardioprotective Effects of Diosgenin. Oxid Med Cell Longev 2017;2017:1-9.

16 Ou-Yang S-H, Jiang T, Zhu L, et al. Dioscorea nipponica Makino: a systematic review on its ethnobotany, phytochemical and pharmacological profiles. Chem Cent J 2018;12:57.

17 Lim S, Eckel RH. Pharmacological treatment and therapeutic perspectives of metabolic syndrome. Rev Endocr Metab Disord 2014;15:329-41.

18 Wang TZ, Huo QP, Wang B, et al. Synergistic effects of Yiqi Huazhuo Gushen herbal formula and valsartan on metabolic syndrome complicated with microalbuminuria. Tropical Journal of Pharmaceutical Research 2019;18:101-8.

$19 \mathrm{Yu}$ X, Xu L, Zhou Q, et al. The efficacy and safety of the Chinese herbal formula, JTTZ, for the treatment of type 2 diabetes with obesity and hyperlipidemia: a multicenter randomized, positivecontrolled, open-label clinical trial. Int J Endocrinol 2018;2018:1-11.

20 Lian J, Shi Y, Wang L, et al. Clinical observation on effect of Yitangkang treating MS with IGR from the BMI levels. Journal of Liaoning University of Traditional Chinese Medicine 2012;14:27-30.

21 Chen L. The clinical curative effect of He ye Jiang Zhi decoction of patients with metabolic syndrome and the effect of circulating ACE and Angll. Hangzhou, China: Zhejiang Chinese Medical University, 2014.

22 Wang $\mathrm{H}$. Effects of He ye Jiang Zhi decoction on the expressions of insulin resistance and inflammatory factor $C$-reactive protein in patients with metabolic syndrome. Hangzhou, China: Zhejiang Chinese Medical University, 2013.

23 Huang P. Clinical study on the effect of resolving phlegm and removing blood stasis on body fat rate of metabolic syndrome with phlegm and blood stasis. Fouzhou, China: Fujian University of Chinese Medicine, 2019.

24 Yang Y. The clinical research of Huayufuyuan capsule to intervene the level of serum inflammatory factors of patients with metabolic syndrome. Nanjing, China: Nanjing University of Chinese Medicine, 2013.

25 Wang T-Z, Huo Q-P, Fu X-D, et al. [Treating Type 2 Diabetes Mellitus Patients Complicated with Metabolic Syndrome by Benefiting Qi Dissolving Method]. Zhongguo Zhong Xi Yi Jie He Za Zhi 2016;36:1065-71.

26 Zhang X. The study on the efficacy and safety of Sanhuangdanshen decoction treating metabolic syndrome (pattern of Phlegmdampness stasis syndrome. Chengdu, China: Chengdu University of Traditional Chinese Medicine, 2014. 
27 Li Q, Zou D, Zhu Z, et al. Treatment of Tangzhiping for metabolic syndrome. Chinese Journal of Experimental Traditional Medical formulae 2012;18:287-90.

28 Ji F. Summary of Professor Wang Wenyou's academic thought and clinical experience and clinical study on metabolic syndrome from Shaoyang. Beijing, China: Beijing University of Chinese Medicine, 2017.

29 Fan Y. Clinical research on treatment of Wulin power on metabolic syndrome. Guangzhou, China: Guangzhou University of Chinese Medicine, 2012.

30 Zhang G, Yi X, Yin L, et al. Clinical effect of Yangyin Jiangya capsule combined with Jiangzhuo Quyu granule in treatment of hypertension with metabolic syndrome. Chinese Journal of Experimental Traditional Medical formulae 2016;22:171-5.

31 Wang $\mathrm{H}$. Clinical observation on effect of Chinese compound prescription Jiangtang pill treating MS with IGR from the age levels. Shenyang, China: Liaoning University of Traditional Chinese Medicine, 2012.

32 Chen X. Effects of traditional Chinese medicine combined with general lifestyle interfering the patients with impaired glucose tolerance combined metabolic syndrome (Qi and Yin deficiency syndrome). Beijing, China: Beijing University of Chinese Medicine, 2011.

33 Liu J, Cui J. Clinical observation on effect of self-made warming spleen and reinforcing healthy Qi decoction. Tianjin Pharmacy 2017;29:31-4.

34 Yu X, Xu L, Zhou Q, et al. The efficacy and safety of the Chinese herbal formula, JTTZ, for the treatment of type 2 diabetes with obesity and hyperlipidemia: a multicenter randomized, PositiveControlled, open-label clinical trial. Int J Endocrinol 2018;2018:1-11.

35 Tong X, Xu J, Lian F, et al. Structural alteration of gut microbiota during the amelioration of human type 2 diabetes with hyperlipidemia by metformin and a traditional Chinese herbal formula: a multicenter, randomized, open label clinical trial. mBio 2018;9:e02392-17.

36 Zhang Y, Kishi H, Kobayashi S. Add-On therapy with traditional Chinese medicine: an efficacious approach for lipid metabolism disorders. Pharmacol Res 2018;134:200-11.
37 Sui Y, Zhao HL, Wong VCW, et al. A systematic review on use of Chinese medicine and acupuncture for treatment of obesity. Obes Rev 2012;13:409-30.

38 Azushima K, Tamura K, Haku S, et al. Effects of the oriental herbal medicine Bofu-tsusho-san in obesity hypertension: a multicenter, randomized, parallel-group controlled trial (ATH-D-14-01021.R2). Atherosclerosis 2015;240:297-304.

39 Jadad AR, Moore RA, Carroll D, et al. Assessing the quality of reports of randomized clinical trials: is blinding necessary? Control Clin Trials 1996;17:1-12.

40 Review Manager (RevMan) [Computer program]. Version 5.3. Copenhagen, Denmark: The Nordic Cochrane Centre, The Cochrane Collaboration, 2014

41 Alberti KG, Zimmet PZ, Definition ZPZ. Definition, diagnosis and classification of diabetes mellitus and its complications. Part 1: diagnosis and classification of diabetes mellitus provisional report of a who consultation. Diabet Med 1998;15:539-53.

42 Balkau B, Charles MA. Comment on the provisional report from the who consultation. European group for the study of insulin resistance (EGIR). Diabet Med 1999;16:442-3.

43 Expert Panel on Detection, Evaluation, and Treatment of High Blood Cholesterol in Adults. Executive summary of the third report of the National cholesterol education program (NCEP) expert panel on detection, evaluation, and treatment of high blood cholesterol in adults (adult treatment panel III). JAMA 2001;285:2486-97.

44 Einhorn D, Reaven GM, Cobin $\mathrm{RH}$, et al. American College of endocrinology position statement on the insulin resistance syndrome. Endocr Pract 2003;9:237-52.

45 Alberti KGMM, Zimmet P, Shaw J, et al. The metabolic syndrome--a new worldwide definition. Lancet 2005;366:1059-62.

46 Joint Committee for guideline revision. 2016 Chinese guidelines for the management of dyslipidemia in adults. $J$ Geriatr Cardiol 2018;15:1-29.

47 Chinese Diabetes Society Metabolic Syndrome Study Group. Chinese diabetes Society recommendation on metabolic syndrome. Chin J Diabetes 2004;12:156-61. 